\title{
OLED from solution-processed crystalline poly(triazine imide)
}

\author{
David Burmeister, ${ }^{[b]}$ Ha Anh Tran, ${ }^{[b]}$ Johannes Müller, ${ }^{[c]}$ Michele Guerrini, ${ }^{[\mathrm{d}]}$ Caterina Cocchi, ${ }^{[c, d]}$ Julian \\ Plaickner, ${ }^{[e]}$ Michael J. Bojdys*[a,b]
}

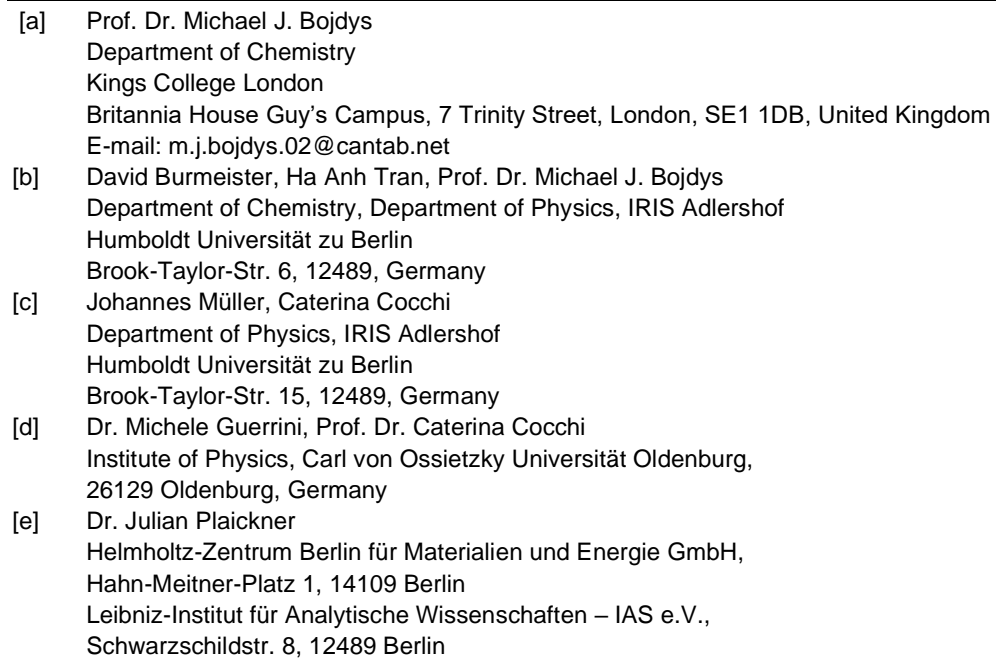

Supporting information for this article is given via a link at the end of the document.((Please delete this text if not appropriate))

\begin{abstract}
Crystalline semiconducting carbon nitrides are chemically and physically resilient, consist of earth abundant elements, and can be exfoliated into 2D atomically thin layers. In particular, poly(triazine imide) (PTI) is a highly crystalline semiconductor, and though no techniques exist to date that enable synthesis of macroscopic monolayers of PTI, it is possible to study it in thin layer device applications that are compatible with its polycrystalline, nanoscale morphology. In our study, we find that the by-product of conventional $\mathrm{PTI}$ synthesis is a C-C carbon rich phase that is detrimental for charge transport and photoluminescence. An optimised synthetic protocol yields a PTI material with an increased quantum yield, enabled photocurrent and electroluminescence. In addition, we report that protonation of the $\mathrm{PTI}$ structure happens preferentially at the pyridinic nitrogen atoms of the triazine $\left(\mathrm{C}_{3} \mathrm{~N}_{3}\right)$ rings, is accompanied by exfoliation of PTI layers, and contributes to increases in quantum yield and exciton lifetimes. This study describes structure-property relationships in PTI that link (i) the nature of defects, their formation, and how to avoid them with (ii) the optical and electronic performance of PTI. On the basis of our findings, we create an OLED prototype with PTI as the active, metal-free material, and we lay the foundations for device integration of solution-processable graphitic carbon nitride dispersions in semiconductor devices.
\end{abstract}

\section{Introduction}

Interest in atomically thin 2D materials skyrocketed with the isolation of graphene sheets from graphite parent crystals and the realisation that these sheets can be exploited in optoelectronic devices. ${ }^{[1]}$ The extraordinarily high charge carrier mobility in graphene of up to $200,000 \mathrm{~cm}^{2} \mathrm{~V}^{-1} \mathrm{~s}^{-1}$ is a result of the changed electronic structure of the atomic layer when compared to the bulk material. ${ }^{[2]}$ The high mobility of semimetallic graphene comes at the price of an absent "off state" that has foreclosed its application in transistors. ${ }^{[3,4]}$ Strategies to open up graphene's bandgap revolve around the introduction of heteroatoms and structural defects in diffusion-limited or statistical post-processing as well as nanostructuring. ${ }^{[5-7]}$ However, none of these methods are scalable and they do not yield products able to compete with silicon based devices to date. ${ }^{[8]}$

Crystalline organic 2D materials that contain predictable "deletions" (i.e. pores) and heteroatoms by design are researched to make up for the missing bandgap of graphene. Most notably transition metal dichalcogenides, graphene derivatives and designer covalent organic frameworks (COFs) compete to be the next generation semiconductor. ${ }^{[8-12]}$

Graphitic carbon nitrides combine two important characteristics: (i) they are overall $\mathrm{sp}^{2}$-hybridised and covalently bonded, and (ii) they consist of earth abundant, light elements (i.e. they are metal free). ${ }^{[13,14]}$ Poly(triazine imide) (PTI-MX) is a layered intercalation compound obtained from alkali halide salt melts and can be exfoliated to monolayers. ${ }^{[15]}$ Even though the material has high environmental stability, crystallinity, blue photoluminescence, and has found application as semiconductor in photo- and electrocatalyst, it has found little to no use in optoelectronic devices up to this point. ${ }^{[16-18]}$ In this work, (i) we elucidate the impact of different polycondensation conditions on the chemical makeup of PTI and its optical and electronic performance, (ii) we monitor the material quality at microscopic and macroscopic scales, (iii) we assemble photoconductor and organic light emitting diode (OLED) devices based on PTI, and (iv) we look at the effect of protonation on the electronic structure of PTI comparing results from theoretical calculations with observed optical spectra. The results of this study provide guidelines for improving the optical and electronic properties of organic layered materials; especially ones that incorporate triazine $\left(\mathrm{C}_{3} \mathrm{~N}_{3}\right)$ subunits. 
lonothermal condensation-polymerisation of dicyandiamide was first performed by Bojdys et al., and later, Wirnhier et al. solved the structure of the product as poly(triazine imide). ${ }^{[19,20]}$ While the structure of this material was examined in detail, the electronic properties of this system have not been studied thoroughly yet. ${ }^{[17,19,21,22]}$ We find that the reduction of the temperature increases the structural order and preserves a desired CN-ratio. This enables us to harness the semiconducting properties in simple prototype devices. To access quality markers for the obtained product we applied powder X-ray diffraction (PXRD), scanning electron microscopy (SEM), X-ray Photoelectron spectroscopy (XPS), Fourier-transform infrared spectroscopy (FT-IR), UV-Raman, solid state nuclear magnetic resonance spectroscopy (ssNMR), UV-Vis absorption spectroscopy (UV-Vis), photoluminescence, and photoluminescence excitation spectroscopy (PL, PLE) as well as quantum yield and lifetime measurements.

The observations of this work also are of explanatory value (i) for the wider organic materials community studying the effects of defects in conjugated polymer semiconductors and (ii) in the context of wide-spread studies into the photocatalytic activity of PTI. ${ }^{[23]}$

\section{Results and Discussion}

\section{Structural characterisation of obtained products}

The starting point for this work is the ionothermal synthetic protocol for PTI that has not been altered since its first reports. ${ }^{24-}$ 26] The significant parameters for polycondensation reactions are the reaction temperature $\left(600^{\circ} \mathrm{C}\right)$ and the reaction time $(12 \mathrm{~h})$. The product is characterized as a dark brown powder (Figure 1a, center). ${ }^{[2]}$ The material shows no visible photoluminescence under $375 \mathrm{~nm}$ UV-light (Figure S1). Higher luminescence can typically be accessed only by dispersing the material in water/methanol/DMSO. ${ }^{[26]}$ Hence, it is often argued that the emission of the material in its initial graphitic state is quenched by $\pi-\pi$ stacking. Instead of excitation and radiative emission, $\pi-\pi$ quenching leads to photo induced electron transfer to a neighboring $\pi$-system. This inhibits effective recombination of the electron hole pair in a radiative process.

We report that synthesis at a temperature of $550^{\circ} \mathrm{C}$ and extended reaction times of $48 \mathrm{~h}$ yields an almost colorless product (Figure 1a, left) with visible photoluminescence when irradiated with UV-light. The hitherto employed harsh reaction conditions $\left(600{ }^{\circ} \mathrm{C}, 12 \mathrm{~h}\right)$ are at the onset of carbonisation of the material and introduce carbonaceous contaminants that darken the color of PTI. ${ }^{[28]}$ Qualitative and quantitative XPS analysis of the C1s core region shows an intense peak at $285 \mathrm{eV}$ and confirms the presence of C-C environments (adventitious C1s, C-C peak at $284.8 \mathrm{eV}$ ) (Figure 1b). ${ }^{[29]}$ This contribution is often assigned to adventitious carbon. ${ }^{24,30,31]}$ a

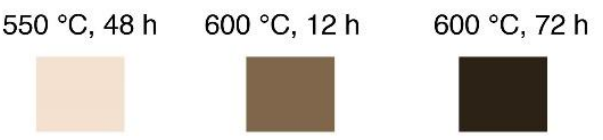

b
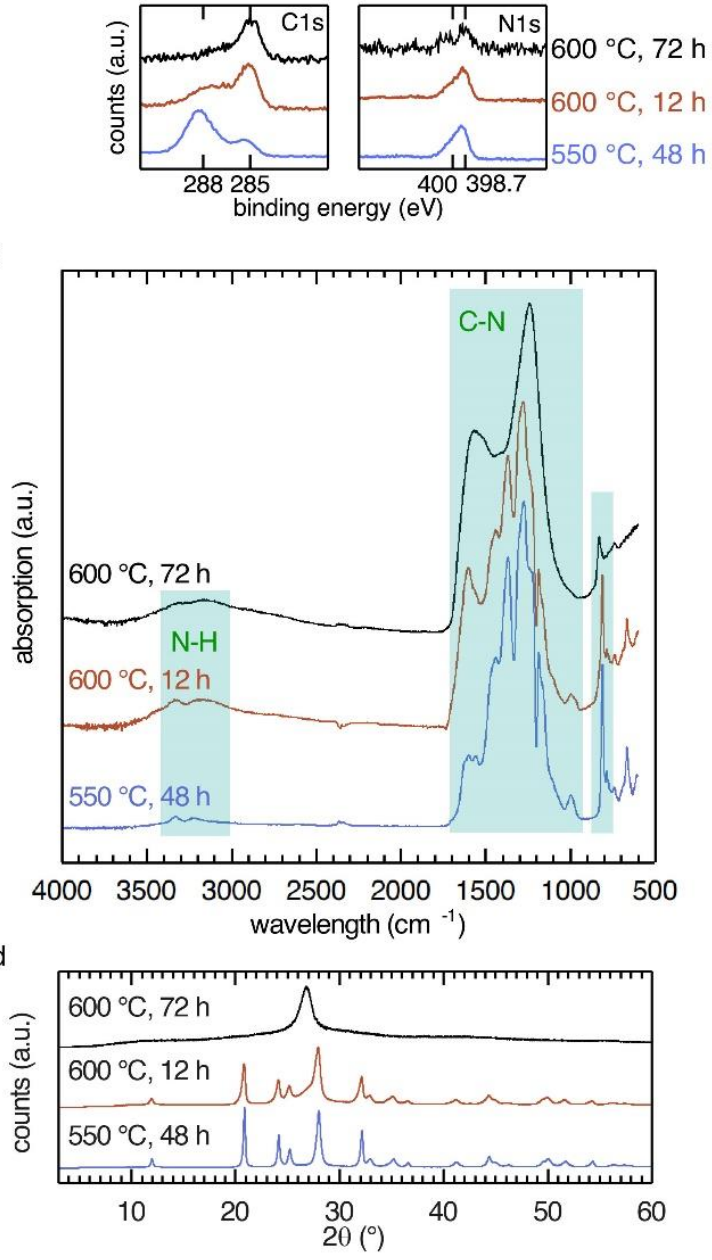

Figure 1. Structural characterization of $\mathrm{PTI}-\mathrm{LiBr}$ materials obtained at different temperatures and reaction times. a) discoloration of $\mathrm{PTI}-\mathrm{LiBr}$ with increasing reaction temperature indicates emergence of carbon rich phase for products formed at $600^{\circ} \mathrm{C}$ b) X-ray photoelectron spectroscopy (XPS) of PTI-LiBr samples. Evaluation of $\mathrm{C} 1 \mathrm{~s}$ and $\mathrm{N} 1 \mathrm{~s}$ core regions confirm the presence of carbon-rich product and loss of nitrogen at $600^{\circ} \mathrm{C}$. C) Fourier transform infrared (FT-IR) spectra of $\mathrm{PTI}-\mathrm{LiBr}$ products show decreased band separation for high reaction temperatures, indicating the loss of structural order. d) Powder X-ray diffraction pattern of product phases. The evolution of a peak at $27^{\circ} 2 \Theta$ for products formed at $600^{\circ} \mathrm{C}$ is interpreted as formation of a disordered carbon rich material next to $\mathrm{PTI}-\mathrm{LiBr}$.

However, at $550{ }^{\circ} \mathrm{C}$ the contribution at $285 \mathrm{eV}$ in the $\mathrm{C} 1 \mathrm{~s}$ spectrum of $\mathrm{PTI}-\mathrm{LiBr}$ is greatly reduced. Since the contribution of adventitious carbon should be similar for all samples, we deduce that part of this contribution comes from a carbon rich phase that coevolved during the synthesis at $600^{\circ} \mathrm{C}$. Looking at the $\mathrm{N} 1 \mathrm{~s}$ region, we observe nitrogen signals from triazine moieties at $398.5 \mathrm{eV}$ and a contribution from the imide- $\mathrm{NH}$ bridges between the triazine units at $400 \mathrm{eV}$ with relative intensities of (3:1) for the 
product from $550{ }^{\circ} \mathrm{C}$ (Figure 1b, right). The product obtained at $550^{\circ} \mathrm{C}, 48 \mathrm{~h}$ approaches the theoretical elemental composition of poly(triazine imide) $\left(\mathrm{C}_{6} \mathrm{~N}_{9} \mathrm{H}_{3}\right)$ of 40:60 C:N most closely (Table 1). Other groups have not reported the presence of this carbon-rich phase at these conditions. Hence it is likely that it was either overlooked or mistaken for a contribution solely from carbon tape used as substrate or adventitious carbon in XPS experiments. An attempt to study the structure of the phase obtained from $600^{\circ} \mathrm{C}$ for $72 \mathrm{~h}$ was conducted by Suter et al. ${ }^{[32]}$

Table 1. X-ray photoelectron spectroscopy (XPS) analysis of atomic percentage of carbon and nitrogen observed in $\mathrm{CN}$ films prepared from different synthetic conditions. The integration of the carbon signal was conducted over all carbon species and not corrected for adventitious carbon.

\begin{tabular}{cc}
\hline $\begin{array}{c}\text { Reaction } \\
\text { conditions }\end{array}$ & C:N at\% XPS \\
\hline $600^{\circ} \mathrm{C}, 72 \mathrm{~h}$ & $87: 13$ \\
$600^{\circ} \mathrm{C}, 12 \mathrm{~h}$ & $81: 19$ \\
$550^{\circ} \mathrm{C}, 48 \mathrm{~h}$ & $49: 51$ \\
\hline
\end{tabular}

To access information about the prevalent bond types and therefore the degree of condensation as well as possible defects, we conducted FT-IR spectroscopy on the products (Figure 1c). FT-IR spectra of the high-temperature products at $600{ }^{\circ} \mathrm{C}$ show a broad peak around $3300 \mathrm{~cm}^{-1}$ in addition to two discernible $\mathrm{NH}$ signals (at 3328 and $3217 \mathrm{~cm}^{-1}$ ). The latter are argued to originate from splitting of the imide $\mathrm{N}-\mathrm{H}$ bond by partial conversion into $\mathrm{N}$ Li. $^{[19]}$ The broad feature is absent in the product obtained at $550{ }^{\circ} \mathrm{C}$. The sharp band around $810 \mathrm{~cm}^{-1}$ is attributed to the out of plane bending mode of the six-membered triazine $\left(\mathrm{C}_{3} \mathrm{~N}_{3}\right)$ ring and is present in all samples. ${ }^{[33-35]}$ Likewise, the material obtained at low temperature has a clearer separation of bands in the $\mathrm{CN}$ stretching region $990-1600 \mathrm{~cm}^{-1}$. There are two valid explanations for these observations: (i) the broad feature around $3300 \mathrm{~cm}^{-1}$ can be attributed to $\mathrm{OH}$ groups attached to the carbonaceous contaminants that are produced at elevated temperatures, and (ii) decreased band separation is indicative of structural disorder in the material obtained at high temperature as a consequence of thermal decomposition. The spectra of samples condensed at $600{ }^{\circ} \mathrm{C}$ for $72 \mathrm{~h}$ resemble those of amorphous $\mathrm{CNH}$-compounds with the dominant infra-red absorption between 1600 and $1300 \mathrm{~cm}^{-1}$ from C-N modes and ring species. ${ }^{[36]}$

PXRD patterns confirm the transition of crystalline PTI- $\mathrm{LiBr}$ into a disordered material at long reaction times and $600{ }^{\circ} \mathrm{C}$ (Figure 1d) Condensation at $550{ }^{\circ} \mathrm{C}$ results in a slight shift in the diffraction pattern towards lower angles corresponding to an increase in plane distances. A conceivable reason is an increased ion loading in the structure obtained at reduced temperature. ${ }^{[37]}$ The same trend of higher product quality at the lower reaction temperature was also found by ssNMR. The $\mathrm{C}^{13}$ spectrum shows the three carbon signals also observed in PTI-LiCl at $\delta=167.0 \mathrm{ppm}$ $162.0 \mathrm{ppm}$ and $158.0 \mathrm{ppm}$ (Figure S2). The bands show increased sharpness in the product condensed at $550{ }^{\circ} \mathrm{C} .{ }^{[19]}$ We examined the sample morphologies by SEM (Figure S3). The morphology of PTI-MX is described as hexagonal platelets and prisms with a basal cross-section of 100-500 nm in literature. ${ }^{[20,38]}$

Further decrease of the reaction temperature to $525^{\circ} \mathrm{C}$ resulted in a product with additional PXRD peaks that could not be attributed to a PTI-type material and, hence, the material was not investigated further (Figure S4). We selected the PTI- $\mathrm{LiBr}$ sample obtained at $550^{\circ} \mathrm{C}(48 \mathrm{~h})$ for further optoelectronic experiments based on its structure and composition.

\section{Optical properties of PTI-LiBr and PTI-IF films}
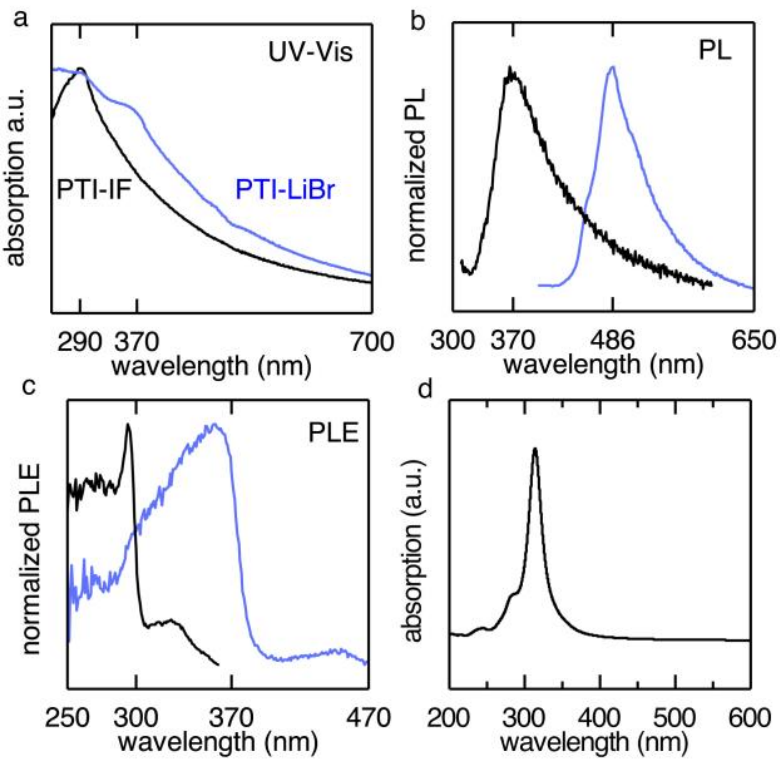

Figure 2. Optical characterisation of $\mathrm{PTI}-\mathrm{LiBr}$ obtained at $550^{\circ} \mathrm{C}$, $12 \mathrm{~h}$ (in blue) and intercalation-free poly(triazine imide) (PTI-IF) (in black) on quartz substrates. a) UV-Vis (transmission) spectrum b) Photoluminescence spectra show emission maxima at $486 \mathrm{~nm}$ for PTI- $\mathrm{LiBr}$ and at $370 \mathrm{~nm}$ for PTI-IF. c) Photoluminescence excitation spectra of PTI- $\mathrm{LiBr}$ (excited at $370 \mathrm{~nm}$ ) and PTI-IF (excited at $300 \mathrm{~nm}$ ). d) Absorption spectrum of a monolayer of PTI-IF computed from time-dependent DFT.

The decreased carbon contamination allows us to measure UVVis of PTI-LiBr dispersions (Figure 2a, blue plot). We find two broad features around 370 and $290 \mathrm{~nm}$. The state at $370 \mathrm{~nm}$ can be excited yielding the photoluminescence spectrum shown in Figure 2b (blue plot). The photoluminescence excitation spectrum shows a single maximum at $486 \mathrm{~nm}$ (Figure 2c, blue line). This emissive state is a result of the replacement of hydrogen with lithium at triazine bridging $\mathrm{N}-\mathrm{H}$ units. This defect reduces the conduction band minimum energy accounting for the contraction of the optical gap of this structure. ${ }^{[27]}$ Exciting PTI- $\mathrm{LiBr}$ at around $290 \mathrm{~nm}$ does not yield a new emission band and decreases the emission at $486 \mathrm{~nm}$ drastically (c.f. Figure 3d). Hence, the state that belongs to the absorption at $290 \mathrm{~nm}$ in PTI$\mathrm{LiBr}$ is quenched.

To gain a better understanding of these two states, we prepared an intercalation free PTI (PTI-IF) comprised only of the CNbackbone (SI Experimental). PXRD, FT-IR and UV-Raman of PTIIF are depicted in Figure S5. Removal of the coordinating lithium and bromine ions leads to disorder as can be found comparing the PXRD spectra of PTI-IF to PTI-LiBr. The width of the reflexes is broadened and some peaks are deleted entirely. Comparing FT-IR spectra of PTI-LiBr and PTI-IF we find better band separation in the ion free PTI-IF as was observed by Suter et al. ${ }^{[2]}$ UV-Raman spectra with a $266 \mathrm{~nm}$ excitation source reveal the 
same trend of sharper bands in PTI-IF. The Raman bands associated with breathing modes of triazine are found at $1002 \mathrm{~cm}$ ${ }^{1}$ (weak) and $685 \mathrm{~cm}^{-1}$ (weak) in PTI-LiBr and $983 \mathrm{~cm}^{-1}$ (strong) and $685 \mathrm{~cm}^{-1}$ (weak) in PTI-IF. ${ }^{[32,39]}$ Broad contributions in both samples at $1300 \mathrm{~cm}^{-1}$ (broad, weak) and $1630 \mathrm{~cm}^{-1}$ (strong) can be assigned to the so called $D$ and $G$ bands due to breathing modes in $\mathrm{sp}^{2}$ bonded rings and $\mathrm{CN} \mathrm{sp}^{2}$ bond stretching in rings and chains respectively. ${ }^{[36]}$ The presence of a sharp, strong Raman band at $1088 \mathrm{~cm}^{-1}$ in PTI- $\mathrm{LiBr}$ samples indicates a carbonate species. ${ }^{[40]}$ The lithium defect can undergo hydrolysis to form $\mathrm{LiOH}$ in presence of water. This is the known reason for the basic character of PTI-MX in water as reported in literature. ${ }^{[41]}$ $\mathrm{LiOH}$ is a known $\mathrm{CO}_{2}$ absorber. ${ }^{[42]}$ We propose that under environmental conditions (in presence of water and $\mathrm{CO}_{2}$ ) $\mathrm{LiOH}$ can form at the surface of the crystallites which in turn can absorb $\mathrm{CO}_{2}$ leading to the presence of lithium carbonate species. Hence, in PTI-IF this Raman band is absent.

Optical characterization by UV-Vis spectroscopy reveals that by removing most of the ions in the structure, we lose the absorption band at $370 \mathrm{~nm}$, as expected, since this state is the result of $\mathrm{Li}$ defects. Absorption at $290 \mathrm{~nm}$ is still prevalent and, hence, it is assigned to the PTI-conjugated backbone. This observation matches well with the theoretical predictions for an intercalation free PTI (see Figure 2d). The result of this calculation shows that PTI-IF layers have a single bright absorption band at around $320 \mathrm{~nm}$, in good agreement with the experimental results, considering the necessary simplifications applied to the model structure adopted in the simulation (see Figure S7,S8) The absence of emission from the $290 \mathrm{~nm}$ state in PTI- $\mathrm{LiBr}$ could be due to the presence of the $\mathrm{Li}$ defects acting as a deep trap. Excitons formed in the organic backbone will recombine primarily at these energetically favorable defect sites. A similar effect can be observed for keto-defects in polyfluorene backbones. ${ }^{[43]}$

It is not possible to determine the quantum yield of PTI samples obtained at $600{ }^{\circ} \mathrm{C}$ because of strong absorption from the accompanying carbon-rich phase. The quantum yield of films cast from the $550{ }^{\circ} \mathrm{C}$ product equals $0.4 \%$. Optical images comparing products obtained at $550^{\circ} \mathrm{C}$ and $600{ }^{\circ} \mathrm{C}$ illuminated with a $375 \mathrm{~nm}$ LED can be accessed in the SI (Figure S1).

\section{Effect of protonation on the optical states of $\mathrm{PTI}-\mathrm{LiBr}$}

In a first set of experiments, we collected UV-Vis spectra of PTI$\mathrm{LiBr}$ suspended in water at different concentrations of $\mathrm{HCl}$ (Figure 3a). Without $\mathrm{HCl}$ we observe the absorption of the lithium defect states at around $370 \mathrm{~nm}$ and the broad absorption of the conjugated PTI-backbone at around $290 \mathrm{~nm}$. By increasing the $\mathrm{H}_{3} \mathrm{O}^{+}$concentration, two effects can be identified. First the absorption band of the organic backbone experiences a slight bathochromic shift from 290 to $300 \mathrm{~nm}$. Moreover, a new absorption band at $260 \mathrm{~nm}$ arises. There are two possible sites of protonation in the PTI- $\mathrm{LiBr}$ structure: (i) at the triazine nitrogen (Figure 3b, ii), and at the imide nitrogen (Figure 3b, iii). Protonation at the triazine nitrogen compares best with the observed UV-Vis spectra, and accounts for the bathochromic shift and the manifestation of a new band at $260 \mathrm{~nm}$ (compare Figure 3b, i and Figure 3b, ii). Our first principles calculations predict that the triazine protonated structure is more stable than the imide bridge protonated structure. Based on this finding, we assume that the triazine protonation is prevalent. The calculated optical absorption spectra of the protonated materials (Figure $\mathbf{3 b}$ ) indicate an overall red-shift of the absorption peaks in comparison with PTI-IF. This result is qualitatively in agreement with earlier predictions on analogous model systems. ${ }^{[44]}$ Specifically the red shift of the main peak in combination with the emergence of a second absorption band in the absorption spectrum of triazine protonated PTI (Figure 3b, ii) is considered an effect of the symmetry breaking of the backbone structure upon protonation. The presence of two absorption bands in the system is in good agreement with two corresponding PLE maxima at $260 \mathrm{~nm}$ and $297 \mathrm{~nm}$ of the emission at $330 \mathrm{~nm}$ (Figure 3d and 3e) present in dispersions with $\mathrm{HCl}$ concentrations larger than $10^{-5} \mathrm{M}$ at $260 \mathrm{~nm}$ and $300 \mathrm{~nm}$ (Figure $\mathbf{3 d}$ and $\mathbf{3 e}$ ).
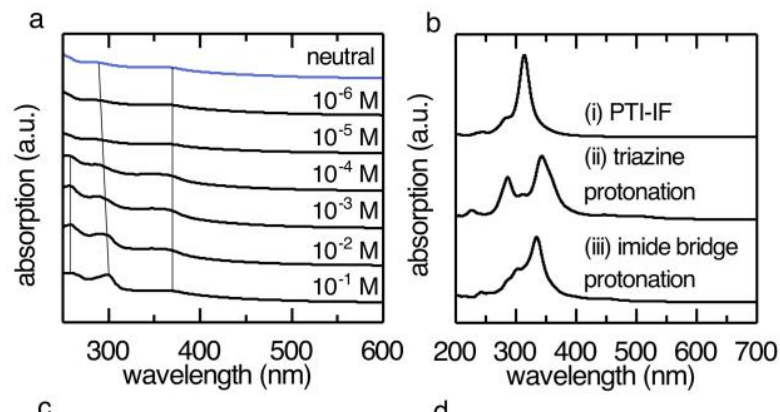

C

d
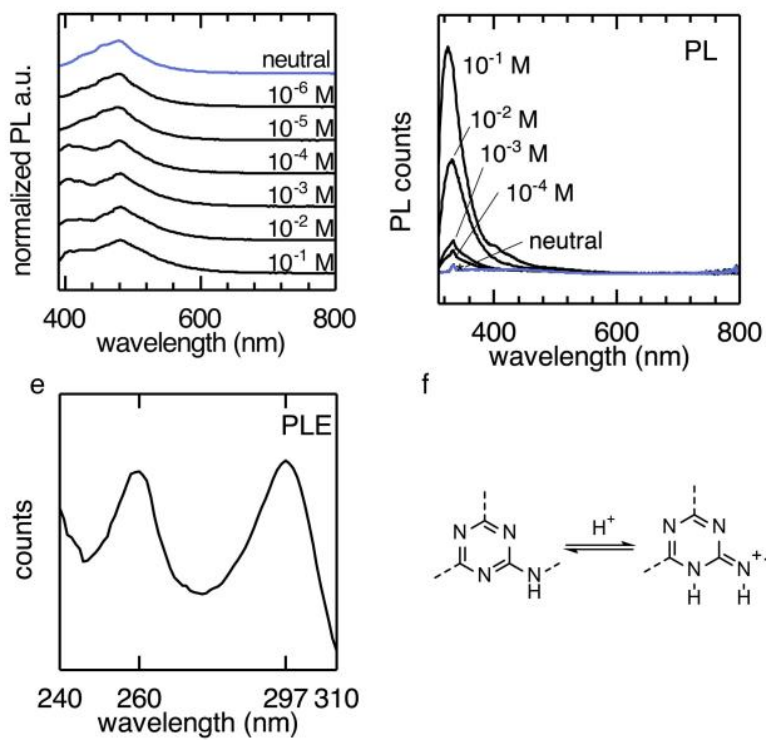

f

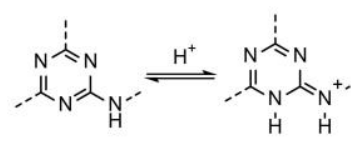

Figure 3. Optical characterisation of PTI-LiBr dispersions in water at different concentrations of $\mathrm{HCl}$. a) UV-Vis (transmission) spectra. b) Time dependent DFT absorption spectrum of (i) a monolayer PTI-IF, (ii) PTI-IF monolayer protonated via $\mathrm{HCl}$ at triazine rings and (iii) PTI-IF monolayer protonated via $\mathrm{HCl}$ at imide bridges. c) Photoluminescence (PL) spectra excited at $370 \mathrm{~nm}$ for PTI-LiBr dispersions with different $\mathrm{HCl}$ concentrations. d) Photoluminescence spectra excited at $300 \mathrm{~nm}$ for PTI-LiBr dispersions with different $\mathrm{HCl}$ concentrations. The Raman band of water is denoted with an asterisk. e) Photoluminescence excitation (PLE) spectrum for emission at $330 \mathrm{~nm}$ at $10^{-2} \mathrm{M} \mathrm{HCl}$. f) Energetically favored protonation at the triazine ring.

It is noteworthy, that for excitation at $300 \mathrm{~nm}$ in dispersions at $10^{-}$ ${ }^{2} \mathrm{M} \mathrm{HCl}$ the quantum yield obtained was $10 \%$ and for excitation at $370 \mathrm{~nm} \mathrm{9 \%}$. The emission of the neutral dispersion at $486 \mathrm{~nm}$ is comparatively low with a quantum yield of $1 \%$ and no emission for excitation at $300 \mathrm{~nm}$. The increased quantum yield is accompanied by an increase in lifetime (neutral $\tau_{1}=0.5 \mathrm{~ns}$ 
$\pm 0.03 \mathrm{~ns}, \tau_{2}=3.6 \mathrm{~ns} \pm 0.13 \mathrm{~ns}, \mathrm{pH} 1 \tau_{1}=1 \mathrm{~ns} \pm 0.05 \mathrm{~ns}, \tau_{2}=4 \mathrm{~ns}$ $\pm 0.10 \mathrm{~ns}$ ). As typical for $\mathrm{CN}$ materials a two exponential fit was applied. ${ }^{[31]}$ The increase in quantum yield and lifetime is attributed to partial exfoliation of the layered PTI structure and, hence, reduced $\pi-\pi$ stacking. Charging of $\mathrm{PTI}$ layers by reduction with sodium naphthalide and stabilization by derivatisation with an $\mathrm{C}_{12}$ alkane was described in literature. ${ }^{[45]} \mathrm{A}$ route via protonation could be a viable alternative.

The photoluminescence band we assign to the $\mathrm{CN}$ backbone protonated at the triazine moiety at $330 \mathrm{~nm}$ is blue-shifted in respect to the emission of PTI-IF at $370 \mathrm{~nm}$ (Figure $3 \mathrm{~d}, 2 \mathrm{~b}$ ).

The changes in the optical spectra coincide with the observation of an equivalence point at $\mathrm{pH} 5$ in the $\mathrm{HCl}$ titration of $\mathrm{PTI}-\mathrm{LiBr}$ dispersions (Figure S6). The pKb extracted from the titration is $\mathrm{pKb}=6.4$. The $\mathrm{pKa}$ value associated with the conjugated acid is $7.6(\mathrm{pKa}=14-\mathrm{pKb})$. The conjugated acid is weaker than the conjugated acid of melamin $(\mathrm{pKa}=5.0) .{ }^{[46]}$ This indicates that the protonated $\mathrm{PTI}-\mathrm{LiBr}$ structure is better stabilized than the triazinecontaining monomer, presumably due to conjugation across the PTI-backbone (Figure 3f). The observed optical states are summarised in Figure $\mathbf{5}$ and compared to the optical gaps predicted by the DFT calculations.

a) PTI-IF
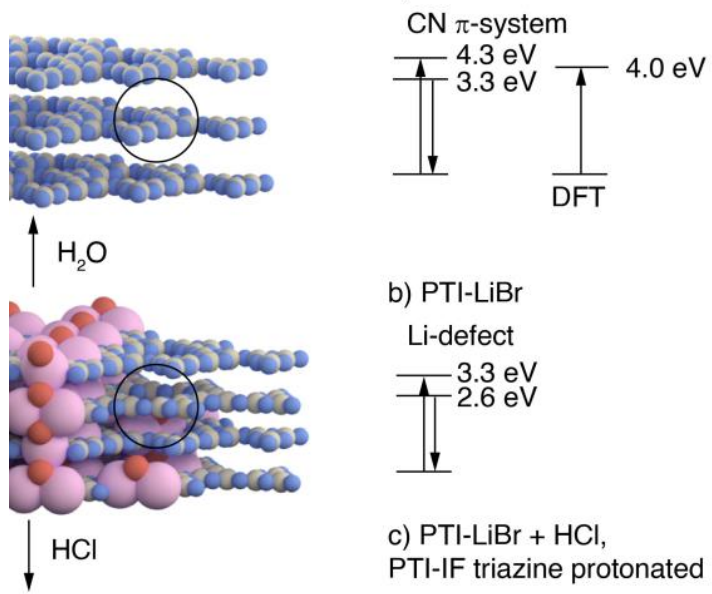

b) PTI-LiBr

Li-defect

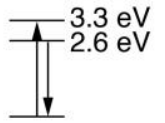

c) $\mathrm{PTI}-\mathrm{LiBr}+\mathrm{HCl}$,

$\mathrm{PTI}$-IF triazine protonated
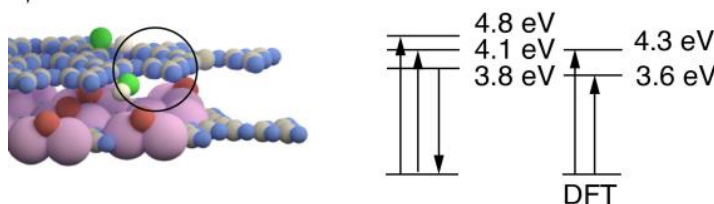

Figure 5. Summary of optical states from DFT, UV-Vis and photoluminescence spectroscopy of dispersions in water of (a) PTI-IF obtained from PTI- $\mathrm{LiBr}$ by Soxhlet extraction of ions, and optical gap of 2D layer of PTI-IF from DFT calculation, (b) partially de-intercalated PTI- $\mathrm{LiBr}$ as obtained from ionothermal synthesis, (c) effects of $\mathrm{HCl}$ at concentrations higher than $10^{-5} \mathrm{M}$ on $\mathrm{PTI}-\mathrm{LiBr}$ dispersions and optical gaps obtained for $2 \mathrm{D}$ layer of triazine protonated PTI-IF sheet by DFT. Arrows pointing upwards denote an absorption process, arrows pointing downwards denote a emission process. Carbon and nitrogen atoms are represented as grey and blue spheres. Hydrogen atoms at the imide nitrogen are not depicted. Lithium and Bromine ions are represented as red and pink spheres. Proton and chloride anion are represented as white and green spheres.

Photoconductor experiments using $\mathrm{PTI}-\mathrm{LiBr}$ were realised on interdigitated indium tin oxide substrates (Figure S9). The conductivity of the material is $410^{-10} \mathrm{~S} \mathrm{~cm}^{-1}$ in the dark and $1.010^{-9} \mathrm{~S} \mathrm{~cm}^{-1}$ in the illuminated state (at $375 \mathrm{~nm}$ ). Photoconductor devices using $\mathrm{PTI}-\mathrm{LiBr}$ undergo changes over the course of the measurement because of the migration of ions. As a consequence, the observed hysteresis in IV-sweeps is dependent on the speed of the sweep (Figure S9). Materials obtained at $600{ }^{\circ} \mathrm{C}$ show no photo-response and can be considered insulating. This is an important observation since it confirms that an increase in structural order and homogeneity in low-temperature PTI samples (as seen in PXRD and FT-IR) and the simultaneous reduction of carbonaceous contaminations translate into better performance of the semiconductor.

We have seen so far that (i) PTI-samples obtained at low temperature conditions $\left(550^{\circ} \mathrm{C}, 48 \mathrm{~h}\right)$ show enhanced electrical properties and that (ii) we are able to process PTI-materials from dispersions. In the following, we successfully prepare a prototype OLED with $\mathrm{Ca} / \mathrm{Al}$ as the cathode, PEDOT:PSS/ITO as anode, and $\mathrm{PTI}-\mathrm{LiBr}$ as the active material (Figure 6a). We observe a relatively low luminance of $2 \mathrm{~cd} \mathrm{~m}^{-2}$ even at high current densities (Figure 6b), that is most likely caused by film inhomogeneities introduced during drop-casting of the PTI- $\mathrm{LiBr}$ layer. Similar to previous observations for $\mathrm{CN}$-films, the electroluminescence maximum of $\mathrm{PTI}-\mathrm{LiBr}$ is red/shifted by $0.5 \mathrm{eV}$ with respect to the PL maximum (Figure 6c). ${ }^{[4]}$ Low-energy defect states are a likely explanation for the red-shift. They can arise from small amounts of carbonaceous contaminants still present in the lowtemperature PTI- $\mathrm{LiBr}$ sample. The presence of carbonaceous contaminants in the low-temperature $\mathrm{PTI}-\mathrm{LiBr}$ sample is corroborated in XPS data that shows a contribution in the C-C bond region (Figure 1b) as well as the still slightly carbon rich $\mathrm{CN}$ ratio (Table 1). 

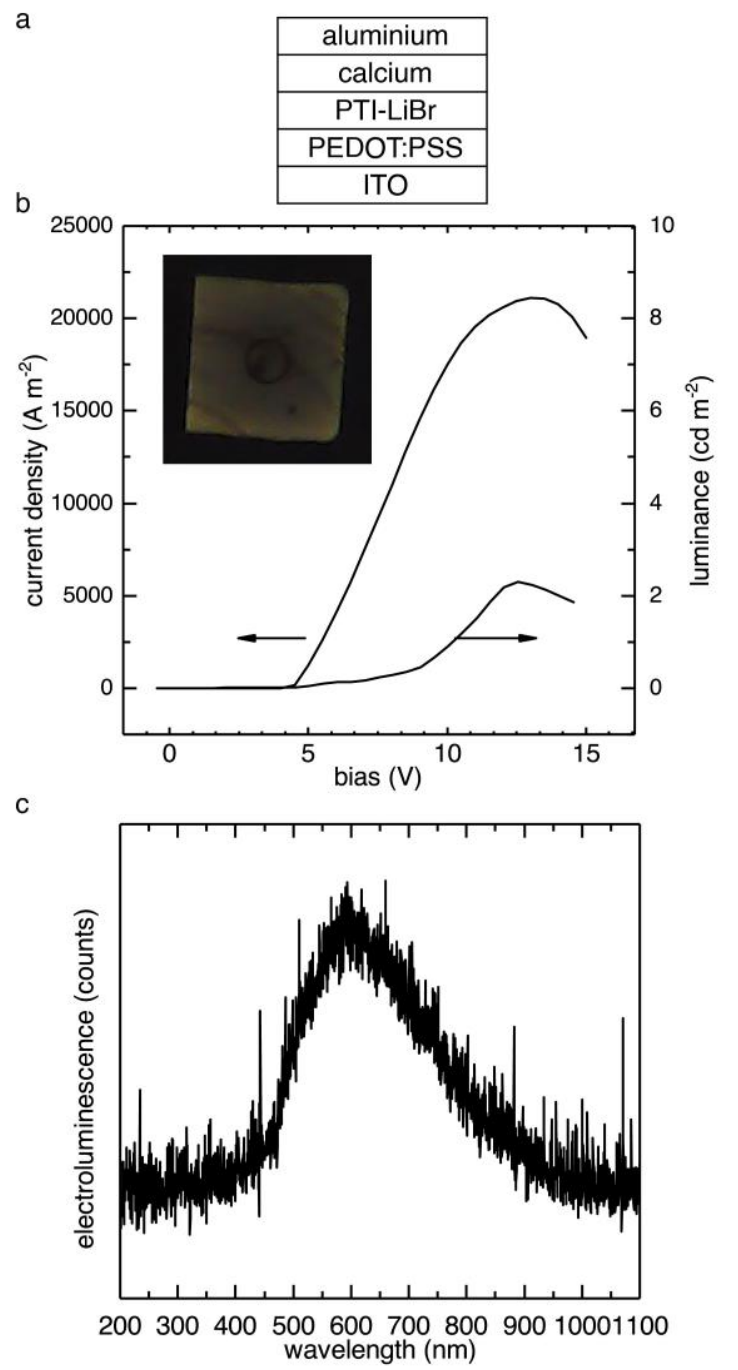

Figure 6. Characterisation of a prototype OLED with $\mathrm{Ca} / \mathrm{Al}$ as the cathode, PEDOT:PSS/ITO anode, and PTI-LiBr as the active material. a) OLED architecture. b) Current density and luminance versus bias with picture of a PTI-LiBr OLED pixel. c) Electroluminescence of the PTI-LiBr OLED.

\section{Conclusion}

We present an improved synthetic protocol for $\mathrm{PTI}-\mathrm{LiBr}$ and highlight the detrimental effects of high synthetic temperatures on the optical and electronic properties of the material. We observe that the hitherto reported synthetic conditions that employ temperatures of $600{ }^{\circ} \mathrm{C}$ (and above) give rise to partial carbonisation of $\mathrm{PTI}-\mathrm{LiBr}$. The formation of these carbonaceous contaminants in $\mathrm{PTI}-\mathrm{LiBr}$ give rise to low-energy defect states that deteriorate charge transfer pathways and absorb photoluminescence. We find that protonation of $\mathrm{PTI}-\mathrm{LiBr}$ happens preferentially at the nitrogen atoms of the triazine $\left(\mathrm{C}_{3} \mathrm{~N}_{3}\right)$ moieties. The absorption band of the PTI-backbone is split due to the symmetry breaking upon protonation. Protonation also increased the quantum yield of PTI suspensions up to a factor of 9.This indicates a facile route to partial exfoliation of the $\pi$-stacked PTI- structure in solutions of aqueous acids. Finally, we are able to produce an OLED prototype with $\mathrm{PTI}-\mathrm{LiBr}$ as an active, metal-free material. The maximum of the electroluminescence is red shifted by $0.5 \mathrm{eV}$ in regard to the photoluminescence. This observation is assigned to the presence of trap states. Future use of PTI (and analogous, layered $\mathrm{CN}$-materials) in optical and electronic applications is contingent on (i) further reduction of contributions arising from low-energy defects and (ii) development of methods to access its crystal interfaces as well as PTI monolayers on suited substrates.

\section{Acknowledgements}

The authors are grateful for the collaboration with Dr. Matthias Trunk, Dr. Felix Hermerschmidt, Prof. Dr. Emil List-Kratochvil, Prof. Christoph T. Koch, PhD, Prof. Dr. Norbert Koch, Prof. Dr. Nicola Pinna, Prof. Dr. Phillip Adelhelm, Prof. Dr. Gudrun Scholz, Prof. Dr. Norbert Esser and Prof. Dr. Hans Börner who provided fruitful discussions, lab space and access to instruments. M.J.B. thanks the European Research Council (ERC) for funding under the Starting Grant Scheme (BEGMAT678462). M.G. and C.C. acknowledge funding by the German Research Foundation (DFG), project number 182087777-CRC 951, by the German Federal Ministry of Education and Research (Professorinnenprogramm III) as well as from the State of Lower Saxony (Professorinnen für Niedersachsen). Computational resources were provided by HPC cluster CARL at the University of Oldenburg, funded by the DFG (Project No. INST 184/157-1 FUGG) and by the Ministry of Science and Culture of Lower Saxony.

Keywords: crystalline carbon nitride, layered materials, metal free semiconductor, covalent-organic-framework, ionothermal synthesis 


\section{References}

[1] A. Castellanos-Gomez, Nat. Photonics 2016, 10, 202-204.

[2] K. I. Bolotin, K. J. Sikes, Z. Jiang, M. Klima, G. Fudenberg, J. Hone, P. Kim, H. L. Stormer, Solid State Commun. 2008, 146, 351-355.

[3] C. J. Shearer, A. D. Slattery, A. J. Stapleton, J. G. Shapter, C. T. Gibson, Nanotechnology 2016, 27, 125704

[4] T. Wu, H. Shen, L. Sun, B. Cheng, B. Liu, J. Shen, New J. Chem. 2012, 36, 1385-1391.

[5] J. Kotakoski, A. V. Krasheninnikov, U. Kaiser, J. C. Meyer, Phys. Rev. Lett. 2011, 106, 1-4.

[6] M. Pumera, Z. Sofer, Chem. Soc. Rev. 2017, 46, 4450-4463.

[7] B. S. Jessen, L. Gammelgaard, M. R. Thomsen, D. M. A. Mackenzie, J. D. Thomsen, J. M. Caridad, E. Duegaard, K. Watanabe, T. Taniguchi, T. J. Booth, et al., Nat. Nanotechnol. 2019 , 14, 340-346

[8] D. Burmeister, M. G. Trunk, M. J. Bojdys, Chem. Soc. Rev. 2021, DOI 10.1039/D1CS00497B.

[9] M. Heyl, D. Burmeister, T. Schultz, S. Pallasch, G. Ligorio, N. Koch, E. J. W. List-Kratochvil, Phys. status solidi-Rapid Res. Lett. 2020, 14, 2000408

[10] P. Miró, M. Audiffred, T. Heine, Chem. Soc. Rev. 2014, 43, 65376554.

[11] A. K. Geim, I. V. Grigorieva, Nature 2013, 499, 419-425.

[12] M. S. Lohse, T. Bein, Adv. Funct. Mater. 2018, 28, 1705553.

[13] G. Algara-Siller, N. Severin, S. Y. Chong, T. Björkman, R. G. Palgrave, A. Laybourn, M. Antonietti, Y. Z. Khimyak, A. V. Krasheninnikov, J. P. Rabe, et al., Angew. Chemie - Int. Ed. 2014, 53, 7450-7455

[14] Y. Noda, C. Merschjann, J. Tarábek, P. Amsalem, N. Koch, M. J. Bojdys, Angew. Chemie 2019, 131, 9494-9498.

[15] L. F. Villalobos, M. T. Vahdat, M. Dakhchoune, Z. Nadizadeh, M. Mensi, E. Oveisi, D. Campi, N. Marzari, K. V. Agrawal, Sci. Adv. 2020, 6, 1-9.

[16] X. Jia, Q. Guan, Y. Chen, Y. Wang, Q. Zhao, J. Li, Appl. Surf. Sci. 2019, 492, 879-885.

[17] W. R. Lee, Y. S. Jun, J. Park, G. D. Stucky, J. Mater. Chem. A 2015, 3, 24232-24236.

[18] L. Lin, C. Wang, W. Ren, H. Ou, Y. Zhang, X. Wang, Chem. Sci. 2017, 8, 5506-5511.

[19] E. Wirnhier, M. Döblinger, D. Gunzelmann, J. Senker, B. V. Lotsch, W. Schnick, Chem. - A Eur. J. 2011, 17, 3213-3221.

[20] M. J. Bojdys, J. O. Müller, M. Antonietti, A. Thomas, Chem. - A Eur. J. 2008, 14, 8177-8182.

[21] C. Z. Liao, V. W. H. Lau, M. Su, S. Ma, C. Liu, C. K. Chang, H. S. Sheu, J. Zhang, K. Shih, Inorg. Chem. 2019, 58, 15880-15888.

[22] X. Yan, J. Li, H. Zhou, J. Mater. Sci. Mater. Electron. 2019, 30, 11706-11713.

[23] L. Lin, Z. Lin, J. Zhang, X. Cai, W. Lin, Z. Yu, X. Wang, Nat. Catal. 2020, 3, 649-655.

[24] T. M. Suter, T. S. Miller, J. K. Cockcroft, A. E. Aliev, M. C. Wilding, A. Sella, F. Corà, C. A. Howard, P. F. McMillan, Chem. Sci. 2019, 10, 2519-2528

[25] M. J. Bojdys, J. O. Müller, M. Antonietti, A. Thomas, Chem. - A Eur. J. 2008, 14, 8177-8182.

[26] T. S. Miller, T. M. Suter, A. M. Telford, L. Picco, O. D. Payton, F. Russell-Pavier, P. L. Cullen, A. Sella, M. S. P. Shaffer, J. Nelson, et al., Nano Lett. 2017, 17, 5891-5896.
[27]

8]

[29]

[30]

[31]

[32]

[34]

[35]

[36]

[40]
E. J. McDermott, E. Wirnhier, W. Schnick, K. S. Virdi, C. Scheu, Y. Kauffmann, W. D. Kaplan, E. Z. Kurmaev, A. Moewes, J. Phys. Chem. C 2013, 117, 8806-8812.

H. May, J. Appl. Chem. 1959, 9, 340-344.

S. Evans, Surf. Interface Anal. 1997, 25, 924-930.

A. . Dementjev, A. de Graaf, M. C. . van de Sanden, K. . Maslakov, A. . Naumkin, A. . Serov, Diam. Relat. Mater. 2000, 9, 1904-1907. X. Li, G. Hartley, A. J. Ward, P. A. Young, A. F. Masters, T. Maschmeyer, J. Phys. Chem. C 2015, 119, 14938-14946.

T2] T. Suter, V. Brázdová, K. McColl, T. S. Miller, H. Nagashima, E. Salvadori, A. Sella, C. A. Howard, C. W. M. Kay, F. Corà, et al., J. Phys. Chem. C 2018, 122, 25183-25194.

T. S. Miller, A. B. Jorge, T. M. Suter, A. Sella, F. Corà, P. F. McMillan, Phys. Chem. Chem. Phys. 2017, 19, 15613-15638. B. V. Lotsch, W. Schnick, Chem. - A Eur. J. 2007, 13, 4956-4968. B. Jürgens, E. Irran, J. Senker, P. Kroll, H. Müller, W. Schnick, J. Am. Chem. Soc. 2003, 125, 10288-10300.

A. C. Ferrari, S. E. Rodil, J. Robertson, Phys. Rev. B 2003, 67, 155306.

S. Y. Chong, J. T. A. Jones, Y. Z. Khimyak, A. I. Cooper, A. Thomas, M. Antonietti, M. J. Bojdys, J. Mater. Chem. A 2013, 1, 1102-1107.

L. Lin, Z. Yu, X. Wang, Angew. Chemie - Int. Ed. 2019, 58, 61646175.

N. E. Mircescu, M. Oltean, V. Chis, N. Leopold, Vib. Spectrosc 2012, 62, 165-171.

0] M. H. Brooker, J. Wang, Spectrochim. Acta Part A Mol. Spectrosc. 1992, 48, 999-1008.

K. Schwinghammer, M. B. Mesch, V. Duppel, C. Ziegler, J. Senker, B. V. Lotsch, J. Am. Chem. Soc. 2014, 136, 1730-1733.

P. Zilberman, Acta Medica Marisiensis 2015, 61, 4-6.

E. J. W. List, R. Guentner, P. S. De Freitas, U. Scherf, Mater. Res. Soc. Symp. - Proc. 2003, 734, 231-236.

4] M. Guerrini, E. Delgado Aznar, C. Cocchi, J. Phys. Chem. C 2020, 124, 27801-27810

J. Jia, E. R. White, A. J. Clancy, N. Rubio, T. Suter, T. S. Miller, K. McColl, P. F. McMillan, V. Brázdová, F. Corà, et al., Angew. Chemie Int. Ed. 2018, 57, 12656-12660.

6] Y. H. Jang, S. Hwang, S. B. Chang, J. Ku, D. S. Chung, J. Phys. Chem. A 2009, 113, 13036-13040.

J. Xu, M. Shalom, F. Piersimoni, M. Antonietti, D. Neher, T. J. K.

Brenner, Adv. Opt. Mater. 2015, 3, 913-917. 


\section{Shedding light on poly(triazine imide), a metal-free semiconductor and photoemitter}

Insert graphic for Table of Contents here. ((Please ensure your graphic is in one of following formats))

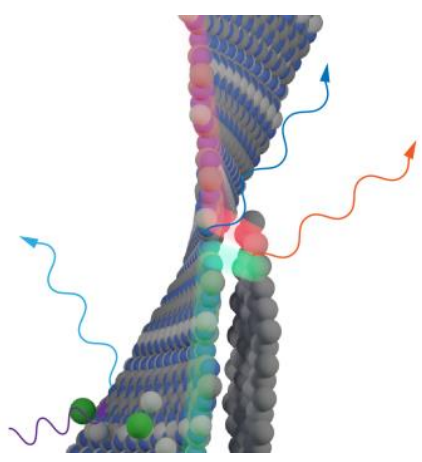

In this contribution, we report the effects of low-energy defects, of charge delocalisation, and of exfoliation on the optical and electronic performance of a layered, metal-free intercalation compound, poly(triazine imide) (PTI). Using an adjusted synthetic protocol, we are able to increase the quantum yield and the photocurrent of the semiconducting PTI. Due to the high structural quality of the product, we were able to investigate structure-property relationships resulting from protonation of PTI's covalent backbone using optical and theoretic methods and to integrate the PTI as the active, metal-free material in an OLED prototype.

Institute and/or researcher Twitter usernames: @IRISAdlershof @mjbojdys @HumboldtUni @davidburmeist @bojdysLAB 